\title{
Realistic Subsurface Anomaly Discrimination Using Electromagnetic Induction and an SVM Classifier
}

\author{
Juan Pablo Fernández, ${ }^{1}$ Fridon Shubitidze, ${ }^{1,2}$ Irma Shamatava, ${ }^{1,2}$ Benjamin E. Barrowes, ${ }^{1,3}$ \\ and Kevin O'Neill ${ }^{1,3}$ \\ ${ }^{1}$ Thayer School of Engineering, Dartmouth College, Hanover, NH 03755, USA \\ ${ }^{2}$ Sky Research, Inc., Hanover, NH 03755, USA \\ ${ }^{3}$ USA ERDC Cold Regions Research and Engineering Laboratory, Hanover, NH 03755, USA
}

Correspondence should be addressed to Fridon Shubitidze, fridon.shubitidze@dartmouth.edu

Received 31 July 2009; Revised 2 December 2009; Accepted 12 February 2010

Academic Editor: Frank Ehlers

Copyright (c) 2010 Juan Pablo Fernández et al. This is an open access article distributed under the Creative Commons Attribution License, which permits unrestricted use, distribution, and reproduction in any medium, provided the original work is properly cited.

\begin{abstract}
The environmental research program of the United States military has set up blind tests for detection and discrimination of unexploded ordnance. One such test consists of measurements taken with the EM-63 sensor at Camp Sibert, AL. We review the performance on the test of a procedure that combines a field-potential (HAP) method to locate targets, the normalized surface magnetic source (NSMS) model to characterize them, and a support vector machine (SVM) to classify them. The HAP method infers location from the scattered magnetic field and its associated scalar potential, the latter reconstructed using equivalent sources. NSMS replaces the target with an enclosing spheroid of equivalent radial magnetization whose integral it uses as a discriminator. SVM generalizes from empirical evidence and can be adapted for multiclass discrimination using a voting system. Our method identifies all potentially dangerous targets correctly and has a false-alarm rate of about $5 \%$.
\end{abstract}

\section{Introduction}

The millions of unexploded ordnance (UXO) strewn about in former battlefields and military practice ranges, of which a significant fraction involve marine or underwater environments, constitute a pressing humanitarian and environmental hazard worldwide [1]. The high false-alarm rates of current sensors and the need to treat every detected anomaly as potentially dangerous result in decontamination costs running into the millions of dollars per acre and extend remediation timescales by decades if not centuries. This state of affairs can only be resolved by developing methodologies that will quickly and reliably identify hazardous items and discriminate them from the morass of innocuous clutter typically found in the field.

The Strategic Environmental Research and Development Program (SERDP) of the United States military supports continuing research that aims to make UXO remediation more efficient and economic. One of SERDP's benchmarks to assess progress is a battery of UXO discrimination blind tests set up in Camp Sibert, a former U.S. Army facility near Gadsden, Alabama. The targets buried in 216 cells-some of which are empty-include unexploded 4.2" mortar shells, mortar explosion byproducts like base plates and partial mortars (i.e., stretched-out half-shells), smaller shrapnel, and unrelated metallic clutter; some examples appear in Figure 1. The different items are distributed in number as shown in Figure 1(d). In 2006, researchers affiliated with Sky Research, Inc. collected data at Camp Sibert using the EM-63, a cart-based step-off time-domain electromagnetic induction (EMI) sensor produced by Geonics Ltd. [2]. In this paper we use those data to demonstrate the performance of a physically complete, fast and clutter-tolerant discrimination approach developed at Dartmouth College and the Cold Regions Research and Engineering Laboratory.

The discrimination process comprises three tasks: localization, characterization, and classification. The secondary field from a visually obscured object depends both on the 


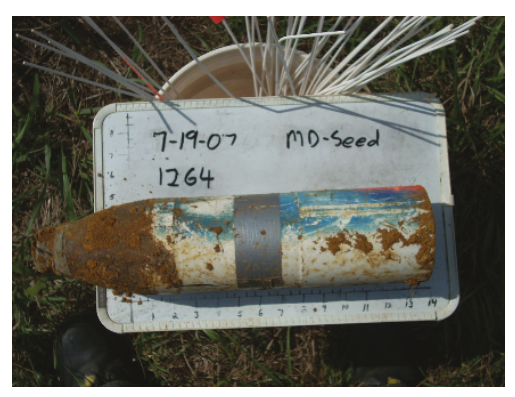

(a) $4.2^{\prime \prime}$ mortar shell

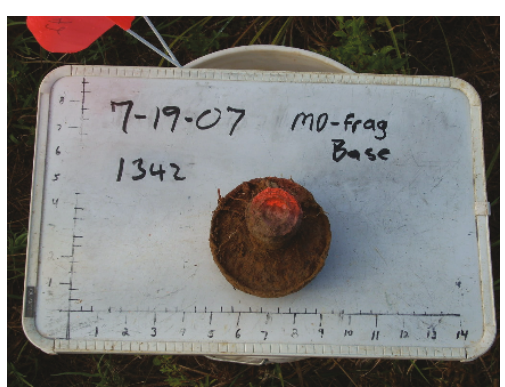

(b) Base plate

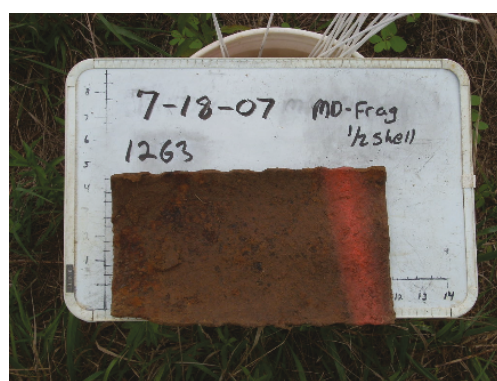

(c) Half-shell

\begin{tabular}{lccc}
\hline Type & Training & Testing & Total \\
\hline UXO & 38 & 34 & 72 \\
Partial & 12 & 23 & 35 \\
Base & 5 & 40 & 45 \\
Scrap & 6 & 25 & 31 \\
Clutter & 4 & 22 & 26 \\
Empty & 1 & 6 & 7 \\
\hline Total & 66 & 150 & 216 \\
\hline
\end{tabular}

(d) Cell contents

FIGURE 1: Representative examples and relative populations of the objects buried at the Camp Sibert cells.

intrinsic features of the target and on its location and orientation relative to the sensor. Attempts to invert simultaneously for positional and intrinsic parameters often result in slow, ill-posed, computationally expensive optimizations that can easily get stuck in local minima. Our method $[3,4]$ clears that hurdle by performing the localization step independently at the outset and then using its results to help in the characterization. This permits a fast and accurate determination of the intrinsic parameters of the model. To classify the targets we feed those parameters to an opensource implementation [5] of a support vector machine (SVM) [6], a machine-learning methodology based on statistical learning theory $[7,8]$ that in the past has been used to perform binary classification [9] and regression [10] and has recently been adapted for multicategory classification [11]. The method has been employed in UXO research, either to classify or regress, in combination with the point-dipole model [1, 12, 13], the Standardized Excitation Approach [14-16], and finite elements $[17,18]$, and has shown to be competitive in its discrimination ability in relation to neural networks [15, 19] and other statistical methods [20, 21].

In a previous paper [22], we studied the Camp Sibert data using the same characterization model in combination with nonlinear least squares for the localization step and both template-matching and a Probability Neural Network for classification. We have already noted [4] that the localization procedure described below results in much better discrimination. The SVM-based classification showcased in this paper improves upon the template-matching used before $[3,4]$ in that it requires less human intervention and is thus faster to run and easier to adapt to other sets of observations. The template-matching procedure made predictions essentially identical to those we report here, perhaps even marginally better, but only after much close monitoring.
SVMs have previously been used for multicategory UXOrelated classification [21], though in that reference the authors' choice of forward model and treatment of positional information differ from ours. While they construct parameter libraries at different locations in order to cancel out the geometric effects and enhance classification, we determine those effects separately; that way not only do we recover critically important information but also obtain parameters whose classification is perhaps easier (and thus faster) to perform and still of high quality.

In summary, our procedure aims to be a powerful and efficient discrimination method for UXO. The precise location and orientation estimates supplied by the so-called HAP technique [23] allow an almost instantaneous determination of an unambiguous time-dependent electromagnetic signature, the total NSMS [24]; this in turn can be distilled further using an empirical decay law [25] whose fitting parameters can be mixed into discriminating features that tend to group in well-separated tight clusters, allowing for clear-cut automated classification using the SVM algorithm.

This paper is organized as follows: in Section 2 we introduce the methods we use to locate and characterize scatterers, in Section 3 we briefly present the principles behind SVM classification, in Section 4 we discuss the results we obtain when we apply the combined procedure to the Camp Sibert data, and in Section 5 we conclude.

\section{A Procedure to Locate and Characterize Obscured Targets}

The eddy currents and magnetic dipoles induced or realigned by an EMI sensor on and inside a scatterer are distributed nonuniformly and tend to concentrate at some particular points. Under certain conditions, the response of the entire 
scatterer can be reproduced to arbitrary precision using a set of responding elementary sources-charges, dipoles, or the like-placed at those singularities $[26,27]$. This consideration underlies the methods that we use to locate and characterize hidden targets.

2.1. A Dipole-Based Method to Estimate Location. The technique we use to locate an obscured target assumes that the whole scatterer responds as a point dipole. The location and orientation of that dipole are then found by exploiting analytic relations involving a dipole field $\mathbf{H}$ and its associated scalar potential $\psi$. (The method originally used the vector potential A as well and has since been dubbed "HAP" [23].) To construct the potential from the field, one distributes elementary sources on an auxiliary planar layer located between the sensor and the object and finds the sources' amplitudes by fitting measured data.

A point dipole of moment $\mathbf{m}$ located at $\mathbf{r}_{d}$ generates at the observation point $\mathbf{r}$ a field

$$
\begin{aligned}
\mathbf{H}(\mathbf{r}) & =\frac{1}{4 \pi\left|\mathbf{r}-\mathbf{r}_{d}\right|^{3}}\left(3 \mathbf{m} \cdot\left(\mathbf{r}-\mathbf{r}_{d}\right) \frac{\left(\mathbf{r}-\mathbf{r}_{d}\right)}{\left|\mathbf{r}-\mathbf{r}_{d}\right|^{2}}-\mathbf{m}\right) \\
& =-\nabla \psi(\mathbf{r})
\end{aligned}
$$

where the scalar potential

$$
\psi(\mathbf{r})=\frac{\mathbf{m} \cdot\left(\mathbf{r}-\mathbf{r}_{d}\right)}{4 \pi\left|\mathbf{r}-\mathbf{r}_{d}\right|^{3}} .
$$

Straightforward algebraic manipulation leads to

$$
\mathbf{H} \cdot \mathbf{r}_{d}=-2 \psi+\mathbf{H} \cdot \mathbf{r}
$$

which provides a least-squares estimate of $\mathbf{r}_{d}$ when evaluated at $N$ distinct observation points:

$$
\left[\begin{array}{ccc}
H_{x}\left(\mathbf{r}_{1}\right) & H_{y}\left(\mathbf{r}_{1}\right) & H_{z}\left(\mathbf{r}_{1}\right) \\
H_{x}\left(\mathbf{r}_{2}\right) & H_{y}\left(\mathbf{r}_{2}\right) & H_{z}\left(\mathbf{r}_{2}\right) \\
\vdots & \\
H_{x}\left(\mathbf{r}_{N}\right) & H_{y}\left(\mathbf{r}_{N}\right) & H_{z}\left(\mathbf{r}_{N}\right)
\end{array}\right]\left[\begin{array}{c}
x_{d} \\
y_{d} \\
z_{d}
\end{array}\right]=\left[\begin{array}{c}
-2 \psi\left(\mathbf{r}_{1}\right)+\mathbf{H}\left(\mathbf{r}_{1}\right) \cdot \mathbf{r}_{1} \\
-2 \psi\left(\mathbf{r}_{2}\right)+\mathbf{H}\left(\mathbf{r}_{2}\right) \cdot \mathbf{r}_{2} \\
\vdots \\
-2 \psi\left(\mathbf{r}_{N}\right)+\mathbf{H}\left(\mathbf{r}_{N}\right) \cdot \mathbf{r}_{N}
\end{array}\right] .
$$

For this particular implementation of the HAP method, it is important to note that the EM-63 sensor measures only the vertical component of the field. To construct the other two components and the scalar potential we assume that the field is produced by a surface distribution of magnetic charge $q\left(s^{\prime}\right)$ spread on a fictitious plane located just below the ground

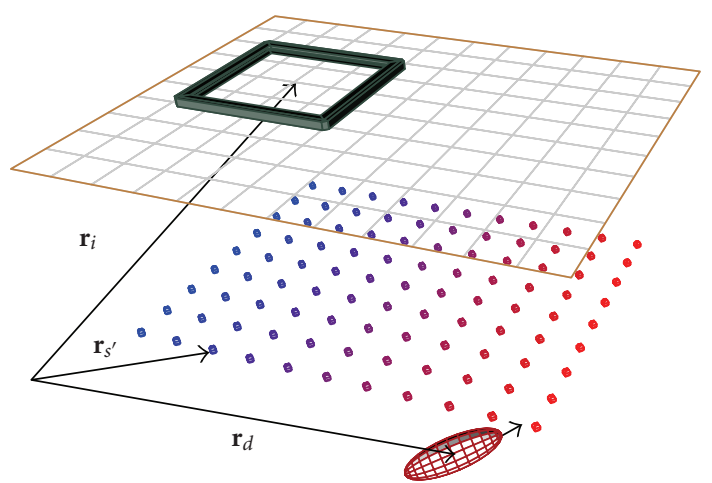

Figure 2: Determining the location and orientation of a buried target. The method assumes the object is a point dipole and exploits an analytic relation between the field measured at $\mathbf{r}_{i}$ and the scalar potential at the same point to find the location $\mathbf{r}_{d}$. The potential is constructed using a layer of equivalent magnetic sources placed between the sensor and the object; $\mathbf{r}_{s^{\prime}}$ is a typical location on the layer.

(see Figure 2). The positions $\mathbf{r}_{s^{\prime}}$ of the sources are fixed and known by construction, and the field can be expressed as the matrix-vector product

$$
H_{z}(\mathbf{r})=\int \frac{q\left(s^{\prime}\right)}{4 \pi} \frac{z-z_{s^{\prime}}}{\left|\mathbf{r}-\mathbf{r}_{s^{\prime}}\right|^{3}} d s^{\prime} \equiv \overleftrightarrow{\mathbf{Z}}_{z} \cdot \mathbf{q}
$$

by employing a quadrature scheme. To determine the array q of charges, one minimizes the difference between model predictions and collected data $\mathbf{H}_{z}^{\text {meas }}$ at a set of known points:

$$
\mathbf{q}=\operatorname{argmin} \frac{1}{2}\left(\overleftrightarrow{\mathbf{Z}}_{z} \cdot \mathbf{q}-\mathbf{H}_{z}^{\text {meas }}\right)^{2}=\left[\overleftrightarrow{\mathbf{Z}}_{z}^{\mathrm{T}} \cdot \overleftrightarrow{\mathbf{Z}}_{z}\right]^{-1}\left[\overleftrightarrow{\mathbf{Z}}_{z}^{\mathrm{T}} \cdot \mathbf{H}_{z}^{\text {meas }}\right]
$$

where each matrix row corresponds to a different measurement point and each column to a subsurface of the underground virtual source layer. The potential is then found from

$$
\psi(\mathbf{r})=\int \frac{q\left(s^{\prime}\right)}{4 \pi\left|\mathbf{r}-\mathbf{r}_{s^{\prime}}\right|} d s^{\prime} \equiv \overleftrightarrow{\mathbf{Z}}_{\psi} \cdot \mathbf{q}
$$

This method and its adaptation to monostatic sensors like the EM-63 used for the Camp Sibert test are discussed in further detail in [23]. One last point is worth reiterating: the HAP method replaces the scatterer with a point dipole, and is thus based on a rather drastic simplification; yet it provides acceptable location estimates because the sources within the target that produce the scattered field tend to concentrate at a set of "scattered field singularities" [26, 27]. The locations of these singularities change at every measurement point, since the primary field of the sensor also changes; the HAP method takes these variations into account and outputs an average location as a result. 
2.2. The Normalized Surface Magnetic Source Model. To encapsulate the electromagnetic signature of a target, we use the fast and robust normalized surface magnetic source (NSMS) model [24]. The particular version we use here associates a scatterer with a surrounding prolate spheroid on which a continuum of radially oriented dipoles are distributed. The strengths of these dipoles-normalized by the normal component of the primary field to take monostaticity into account-are determined as those that best reproduce actual measurements. The composite dipole moment, referred to as the "total NSMS" and denoted by $Q$, varies significantly for different targets but is remarkably consistent for different specimens of the same object.

We divide the spheroid $S$ into patches (or belts to exploit the azimuthal symmetry) and assign

$$
\begin{aligned}
\mathbf{H}^{\mathrm{sc}}(\mathbf{r}) & =\oint_{S} \frac{\Omega\left(s^{\prime}\right)}{4 \pi R_{s^{\prime}}^{3}}\left[\frac{3\left(\widehat{\boldsymbol{\xi}}_{s^{\prime}} \cdot \mathbf{R}_{s^{\prime}}\right) \mathbf{R}_{s^{\prime}}}{R_{s^{\prime}}^{2}}-\widehat{\boldsymbol{\xi}}_{s^{\prime}}\right]\left[\widehat{\boldsymbol{\xi}}_{s^{\prime}} \cdot \mathbf{H}^{\mathrm{pr}}\left(s^{\prime}\right)\right] d s^{\prime} \\
& \equiv \overleftrightarrow{\mathbf{Z}} \cdot \mathbf{\Omega},
\end{aligned}
$$

where $\mathbf{R}_{s^{\prime}}$ is a vector that points from the location $\mathbf{r}_{s^{\prime}}$ of the $s^{\prime}$-th infinitesimal patch on the spheroid to the observation point $\mathbf{r}$ and $\widehat{\boldsymbol{\xi}}_{s^{\prime}}$ is the unit vector normal to the patch. To factor out the particulars of location and orientation we have introduced the normalized surface polarization distribution $\Omega\left(s^{\prime}\right)$. The integral can again be transformed to a matrixvector product via numerical quadrature; each column of $\overleftrightarrow{\mathbf{Z}}$ corresponds to a different source element and each row to a measurement point. The amplitude array $\boldsymbol{\Omega}$ is determined by minimizing in a least-squares sense the difference between measured data with a known object-sensor configuration (as in the case of the Camp Sibert training data) and the predictions of (8).

Once $\Omega\left(s^{\prime}\right)$ is found, one can define a total polarizability by integrating over the whole spheroid. The resulting quantity

$$
Q=\oint_{S} \Omega\left(s^{\prime}\right) d s^{\prime}
$$

a global magnetic capacitance of sorts, has been shown to be intrinsic to the object and can be used, on its own or combined with other quantities, in discrimination processing. Figure 3 shows $Q$ for all 216 Camp Sibert anomalies.

Our analysis of the time dependence of $Q$ has been presented elsewhere $[3,4,22]$ but is worth summarizing here. At early times, where higher frequencies are involved, the skin depth $\delta \propto f^{-1 / 2}$ is small and the induced eddy currents are superficial. As time passes and lower frequencies start to dominate, the currents diffuse into the object, making the late-time response involve the whole volume of the scatterer rather than just its surface. Thus a smaller but solid body like the base plate of Figure 1(b) has a relatively weak early response that dies down slowly, while a large but essentially hollow object like the partial mortar of Figure 1(c) has a strong initial response that decays quickly. The unexploded

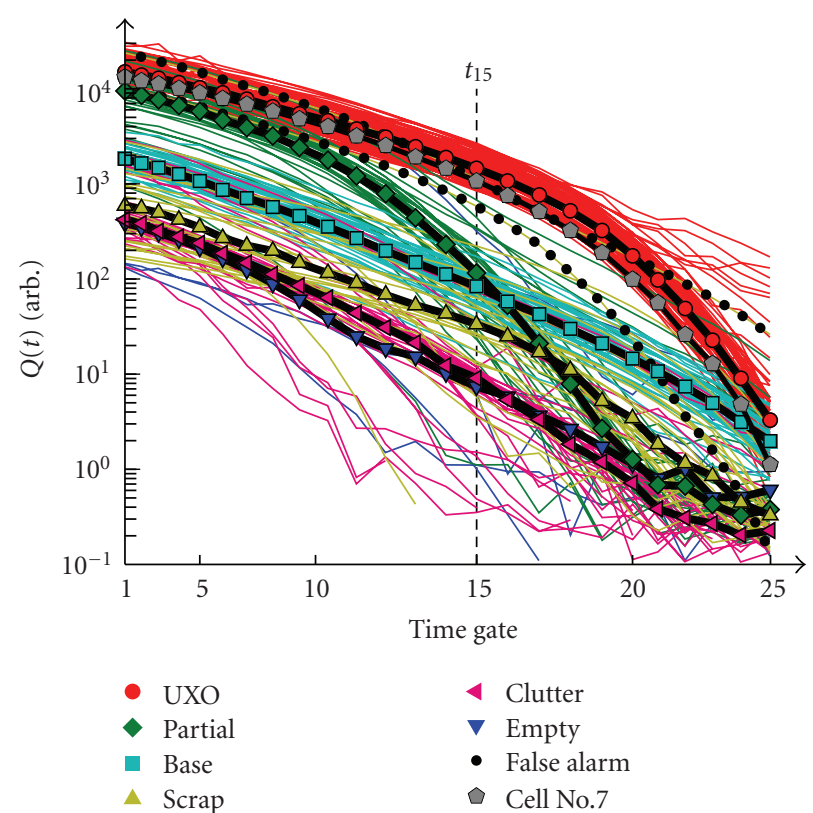

Figure 3: Total NSMS amplitudes $Q(t)$ of the 216 Camp Sibert anomalies as a function of time. The 25 time gates are distributed approximately logarithmically between $191 \mu \mathrm{s}$ and $25 \mathrm{~ms}$. The Qvalues are shown in the same colors and with the same markers as in subsequent figures, with the black line denoting the median $Q$ for each group. The 15th time channel, corresponding to $t=2.72 \mathrm{~ms}$, is highlighted; the ratio $R \equiv Q\left(t_{15}\right) / Q\left(t_{1}\right)$ has been found to be a robust classifier. The gray pentagons correspond to Cell no. 7, which we study in detail in the main text. The thinner dotted lines show the two false alarms from Figure 6, also depicted on Figure 5.

4.2" mortar is large and compact and has a substantial early response that takes a while to die off. Our aim is to use these characteristics of $Q$ to highlight quantitatively the differences between the various targets.

The information contained in $Q$ as a function of the time $t$ can be summarized further by fitting to it an empirical decay law first proposed by Pasion and Oldenburg [25]:

$$
Q(t)=k t^{-\beta} e^{-\gamma t} .
$$

Various combinations of these fitting parameters can be used as inputs to classifier programs, of which the support vector machine (SVM) is an example.

\section{Support Vector Machines for Subsurface Object Classification}

A support vector machine learns from data: when fed a series of answered training examples, it attempts to make sense of them by weighing the available empirical evidence, with no need for an underlying model, and applies this knowledge to make predictions about unseen cases. The examples can be any combination of model parameters expected to contain evidence of the essence of an object. In the simplest instance of binary classification, each $n$ dimensional example $\mathbf{x}_{i}$ has an associated yes/no attribute $y_{i}= \pm 1$; the SVM performs the classification by finding 
a hyperplane that divides the parameter space into two distinct regions, each of which ideally contains points from only one of the categories. During the learning or training process the machine readjusts the hyperplane parameters to accommodate every training vector until it strikes an optimal balance between fitting accuracy and model simplicity. All information about the hyperplane is contained in a subset of the examples-the support vectors that give the method its name-which are then combined to specify a predicting function.

The SVM algorithm uses two different strategies to tackle the nonseparability of realistic data. On one hand, it projects the examples into a space of higher dimensionality by means of a kernel function [28]. The separating surface thus found is flat by construction in the new space but can be curved and even multiply connected in the original. On the other hand, the technique tries to control overfitting-and thus concentrate on essentials rather than on details, resulting in better generalization-by having an adjustable penalty on misclassifications. This penalty is represented by a single scalar parameter, the capacity of the machine [29].

During training, an SVM solves the constrained quadratic optimization problem [30]

$$
\begin{array}{cl}
\max _{\alpha} & \sum_{i} \alpha_{i}-\frac{1}{2} \sum_{i, j} \alpha_{i} y_{i} \mathbf{x}_{i}^{\mathrm{T}} \mathbf{x}_{j} y_{j} \alpha_{j} \\
\text { s.t. } & \sum_{i} \alpha_{i} y_{i}=0, \quad 0 \leq \alpha_{i} \leq C,
\end{array}
$$

whose solution is a vector of coefficients $\alpha_{i}$ that measure the information content of the examples and are nonzero only for the support vectors. The coefficients are prescribed not to exceed the capacity $C$, which limits the influence of potentially problematic points on the final result.

The projection to higher dimensions occurs by substituting the scalar products

$$
\mathbf{x}_{i}^{\mathrm{T}} \mathbf{x}_{j} \longrightarrow K\left(\mathbf{x}_{i}, \mathbf{x}_{j}\right) \equiv \phi\left(\mathbf{x}_{i}\right)^{\mathrm{T}} \phi\left(\mathbf{x}_{j}\right)
$$

for some mapping $\phi(\mathbf{x})$. The function $K$ is the kernel we mentioned earlier. It is not necessary to know $\phi$ to find $K$ : any function that combines two vectors into a scalar and fulfills the (not very restrictive) set of conditions spelled out in Mercer's theorem [31] can be used as a kernel. Some kernels stretch out the examples into the added dimensions in such a way that gaps open up between the examples which permit a flat separating surface to pass through. In this paper, we use the radial basis function (RBF) kernel

$$
K\left(\mathbf{x}_{i}, \mathbf{x}_{j}\right)=\exp \left(-\frac{\left(\mathbf{x}_{i}-\mathbf{x}_{j}\right)^{\mathrm{T}}\left(\mathbf{x}_{i}-\mathbf{x}_{j}\right)}{2 \sigma^{2}}\right),
$$

which surrounds every example with a surface that in a sense "repels" the separating hyperplane. The Gaussian width $\sigma$ is a second adjustable parameter and usually has a scale on the order of the average separation between points. In [32] it was found that polynomial kernels may outperform the RBF kernel in some electromagnetic inverse problems. We find that the linear kernel makes similar predictions and runs faster than the RBF, though the difference in run time is negligible for the number of training data and example features that we use in this study.

Once $\boldsymbol{\alpha}$ is known, the SVM can predict the attribute of an unknown example using the function $[29,33]$

$$
f(\mathbf{x})=\operatorname{sgn}\left(\sum_{i \in \mathrm{sv}} \alpha_{i} y_{i} K\left(\mathbf{x}_{i}, \mathbf{x}\right)\right) .
$$

There are several ways to generalize the SVM procedure to perform multiclass categorization. These have been reviewed in [11], whose authors conclude that the methods more suitable for practical use perform several binary classifications instead of attempting to separate all classes at once. In this work we adopt a one-against-one approach [34] in which the system carries out $\left(\begin{array}{l}k \\ 2\end{array}\right)=k(k-$ 1)/2 optimizations and obtains the same number of decision functions of the form (14). When given an example to predict, the algorithm proceeds by ballot: it evaluates the decision functions one by one on the example and adds a vote to the one category (out of two) in which it is predicted to be. At the end, the example is assigned to the category with the most votes; should there be a tie between two classes, the program arbitrarily selects that with the smallest label.

\section{Results}

4.1. Data Acquisition. The Camp Sibert blind-test data were collected over 216 test cells, each of which was a square plot of side $5 \mathrm{~m}$ and contained at most one anomaly. The targets of interest were $4.2^{\prime \prime}$ mortar shells like the one in Figure 1(a), which were to be discriminated from explosion byproducts represented by the base plates and partial shells of Figures 1(b) and 1(c). Other sites had smaller shrapnel or non-UXO related scrap instead, and a few were essentially empty. We were given the ground truth for 66 of these cases, which we used to build a catalog of expected total NSMS values that were then tested on the 150 other cells. The EM63 took data over 26 channels that span in approximately logarithmic fashion a lapse of time between $180 \mu$ s and $25 \mathrm{~ms}$. In our analysis, we use 25 of these channels, starting with the second. Measurements were taken at grids of between 400 and 700 points that crisscrossed each cell; each grid row was separated some $50 \mathrm{~cm}$ from its neighbors, and within each track the spacing between consecutive measurements was on the order of $5 \mathrm{~cm}$. The EM-63 was always placed $30 \mathrm{~cm}$ above the ground. Figure 4 shows a typical experimental situation, corresponding to Cell no. 7 of the study and containing 668 measurement points.

4.2. Target Location and Characterization. For each data set we run the HAP method of Section 2.1 to locate the target and a fully three-dimensional implementation of the NSMS model of Section 2.2 to characterize it. Consider again the example cell shown on Figure 4. To find the target, we take a fictitious $5 \mathrm{~m} \times 5 \mathrm{~m}$ flat square surface concentric with the 


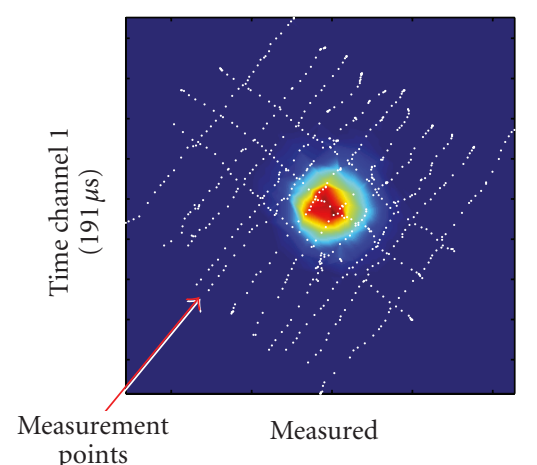

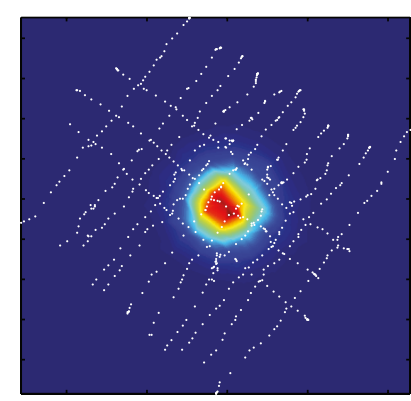

Predicted

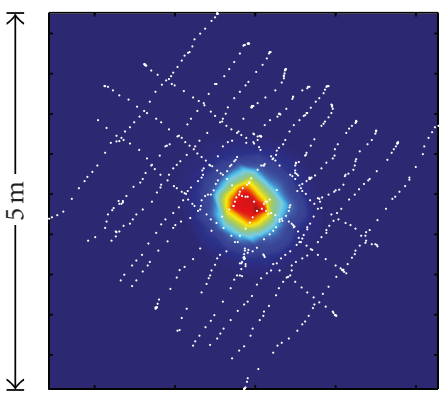

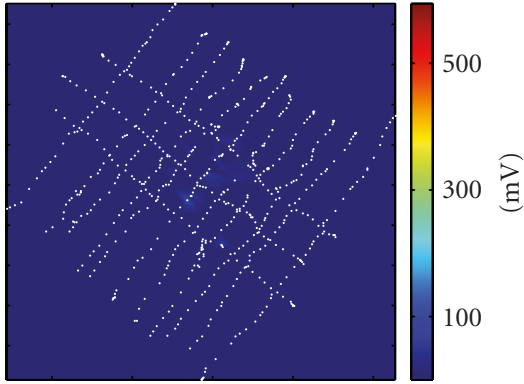

|Measured - predicted $\mid$

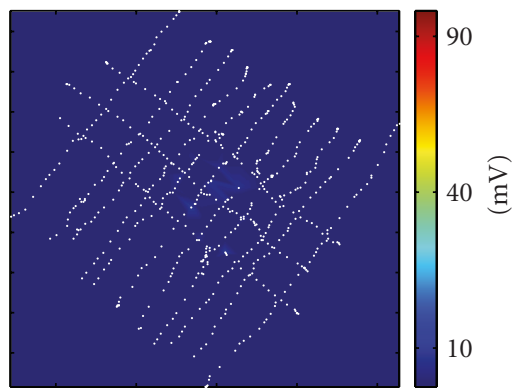

Figure 4: A typical cell from the Camp Sibert EM-63 blind test, no. 7 in this case, consists of a square plot of side $5 \mathrm{~m}$. The white dots show the measurement points distributed on a 668-point grid; the separation between rows is about $50 \mathrm{~cm}$ and that between consecutive points is some $5 \mathrm{~cm}$. The contour plots show the measured (left column) and HAP/NSMS-predicted (middle column) near-field distributions for this cell, as well as the mismatch between the two (right column), for two time gates. The first time channel (top row) is taken $191 \mu$ s after shutdown; the 15th (bottom) is centered at $2.72 \mathrm{~ms}$.

plot and located $30 \mathrm{~cm}$ below the sensor (i.e., at ground level) and divide it into $11 \times 11$ patches, each of which is assumed to contain a magnetic-charge distribution of uniform density. We take the measured field data (as seen for example on the left column of Figure 4) and use (5) to determine $\mathbf{q}$, which in turn allows us to determine $\psi(\mathbf{r})$ using (7) and construct the matrices of (4) to find the location. We do this separately for every time channel and get consistent location estimates from gate to gate, which lends credence to their precision. For the case of Cell no. 7, we obtain a target depth of $55 \mathrm{~cm}$, acceptably close to the ground truth of $60 \mathrm{~cm}$.

To compute the NSMS amplitude $Q(t)$ we surround the target with a prolate spheroid with semiminor axis $a=5 \mathrm{~cm}$ and elongation $e \equiv b / a=4$. This spheroid is divided into 7 azimuthal belts, each of which is assumed to contain a radial-magnetic-dipole distribution of constant density. The spheroid is placed at the location estimated by the HAP method and the orientation given by the dipole moment $\mathbf{m}$ obtained from (2) and (7). With all the pieces in place, we can proceed to apply (8) to find $\boldsymbol{\Omega}$ and (9) to extract $Q(t)$ for the target, which eventually reveals it as the UXO of Figure 5(a). This $Q(t)$ curve appears as a line of pentagons in Figure 3, which also depicts the resulting total NSMS values for the rest of the targets. We determine the Pasion-Oldenburg parameters $k, \beta$, and $\gamma$ for each anomaly by a direct nonlinear least-squares fit of (10) and by linear (pseudo)inversion of its logarithm; both procedures gave consistent results. In general, we obtain good fits to the measured fields [4]; Figure 4 shows that the discrepancy between the actual data and the model prediction runs only to a few percent.
We have previously found $[3,4]$ that the ratio of $Q$ at the 15th time channel to $Q$ at the first time channel, which involves a fixed superposition of $\beta$ and $\gamma$, shows discernible clustering for this particular data set when combined with the third parameter $k$. (The 15th time channel, centered at about $2.7 \mathrm{~ms}$, was chosen because it takes place late enough to show the behavior described above but early enough that all targets still have an acceptable signal-to-noise ratio; nearby time channels produce similar results.) The values of $R \equiv Q\left(t_{15}\right) / Q\left(t_{1}\right)$ for the $4.2^{\prime \prime}$ mortars are particularly well grouped and for the most part noticeably distinct from those of the others, suggesting that this two-dimensional feature space may be used to perform dependable classification. This suggestion is confirmed by our SVM analysis.

4.3. SVM Classification. We use a Gaussian RBF kernel for the SVM analysis. The kernel width turns out not to have much influence on the outcome; we usually set it so that a unit in a typical $x$ - or $y$-axis in a $\log$ plot (Figure 6, say) comprises $100 \Delta$ Gaussian widths, where $\Delta$ is the dimensionality of the feature space. To find the capacity, we train the SVM with a subset of the training data and a given $C$, scramble the training set, and use a new subset of the data for testing. We then vary $C$, setting it to a high value initially and then lowering it, and keep the lowest capacity with which the machine identifies all dangerous items in the test. The procedure is rather ad hoc but effective for the data at hand, given the small sample sizes, the low dimensionality of the feature spaces, and the speed of the 


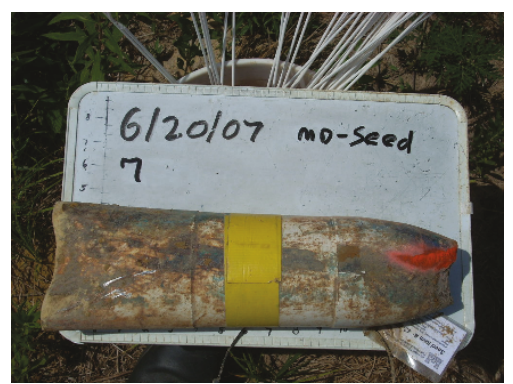

(a) Shell at no. 7

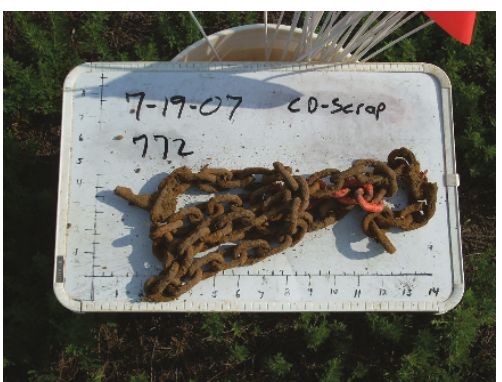

(b) Twisted chain

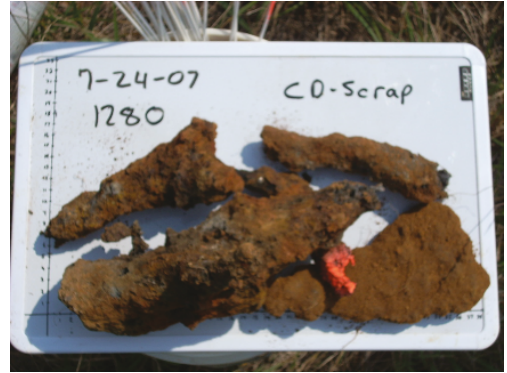

(c) Crumpled debris

Figure 5: (a) Unexploded shell from Cell no. 7, and (b), (c) the two false alarms obtained by the SVM classifier using $k$ and $R$ as discriminators.

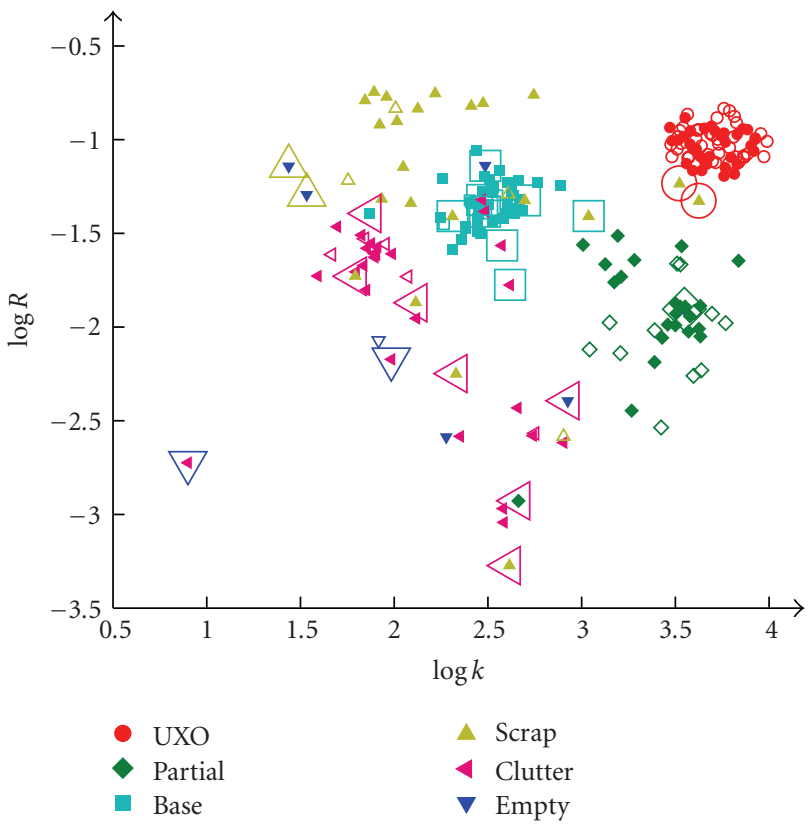

FIGURE 6: Result of the SVM classification for the Camp Sibert anomalies using the logarithms of $k$ and $R \equiv Q\left(t_{15}\right) / Q\left(t_{1}\right)$. The SVM has been trained with capacity $C=10$ and kernel width $\sigma=$ $1 / 200$. The small markers denote the ground truth for both training (hollow) and testing (solid) cells. The larger markers highlight the cases where there is a disagreement between the ground truth and the SVM prediction.

SVM implementation. A more systematic search for $C$ and $\gamma$ using five-fold cross-validation [5] recommends slightly higher capacities that result in identical predictions.

For $R$ and $k$ as features, we find the best SVM performance using $C=10$. The results are displayed (for testing data only) in Table 1 and shown pictorially (for both training and testing) in Figure 6. The matrix element $c_{i j}$ in the table denotes an item of category $i$ that was identified by the SVM as belonging to category $j$; in other words, the rows of this contingency table correspond to the ground truth and the columns to predictions. The small markers in the plot show the ground truth (hollow for training data and filled for the tests), while the large markers point out the items for which the SVM makes wrong predictions. For example, a small yellow upright triangle surrounded by a large cyan square is a piece of scrap (clutter unrelated to UXO) incorrectly identified as a base plate. The UXO, with their high initial amplitudes and slow decay, are clustered at the top right corner. We see that there are only two false alarms (i.e., objects identified with UXO that were in fact something else) and that all potentially dangerous items have been identified correctly.

The false alarms, two pieces of non-UXO clutter, appear on Figures 5(b) and 5(c). They are seen to be similar to the $4.2^{\prime \prime}$ mortars in size and metal content (cf. Figure 5(a)), which makes their $k$ and $R$ values lie closer to the tight UXO cluster than to any other anomaly in Figure 6. Here we note that, as can be seen in Figure 1(d), the training data provided by the examiners was somewhat biased toward UXO, while clutter and scrap samples were underrepresented (this was not the case with the testing data and should not be expected in future tests). If we switch training and testing data in the SVM analysis, we can achieve perfect discrimination without varying the capacity - though in this case we have more training data than tests. This highlights the importance of having a diverse collection of representative samples to use during the training stage.

We can repeat the analysis using other two-dimensional combinations of the Pasion-Oldenburg parameters. Combining $k$ and $\gamma$ yields results similar to those of $k$ and $R$, as Figure 7 and Table 2 show. Figure 8 and Table 3 show the classification resulting from the use of $\beta$ and $\gamma$ as discriminators. The table shows that we can obtain reasonable discrimination, with all the UXO once again correctly identified, but the increased number of false alarms and the very high capacity needed (four orders of magnitude larger than the previous ones) indicate that this combination of parameters may not be optimal and that this machine is prone to overfitting. A glance at the figure shows that the clustering is much less clear-cut than in the previous cases, partly because the range of $\beta$ is rather small. In fact, combining $k$ and $\beta$ greatly reduces the performance, since the small $\beta$-range and the close similarity in $k$ of the UXO and the partial mortars causes an overlap between the two categories that cannot be disentangled. 
TABLe 1: SVM classification of Camp Sibert anomalies using $k$ and $R$ with $C=10$.

\begin{tabular}{|c|c|c|c|c|c|c|c|}
\hline \multirow{2}{*}{\multicolumn{2}{|c|}{$k, R ; C=10$}} & \multicolumn{6}{|c|}{ SVM prediction } \\
\hline & & UXO & Partial & Base & Clutter & Scrap & Empty \\
\hline \multirow{6}{*}{ Ground truth } & UXO & 34 & 0 & 0 & 0 & 0 & 0 \\
\hline & Partial & 0 & 22 & 0 & 1 & 0 & 0 \\
\hline & Base & 0 & 0 & 39 & 1 & 0 & 0 \\
\hline & Clutter & 0 & 0 & 4 & 19 & 0 & 2 \\
\hline & Scrap & 2 & 0 & 3 & 4 & 13 & 0 \\
\hline & Empty & 0 & 1 & 1 & 1 & 2 & 1 \\
\hline
\end{tabular}

TABLE 2: SVM classification of Camp Sibert anomalies using $\gamma$ and $k$ with $C=9$.

\begin{tabular}{|c|c|c|c|c|c|c|c|}
\hline \multirow{2}{*}{\multicolumn{2}{|c|}{$\gamma, k ; C=9$}} & \multicolumn{6}{|c|}{ SVM prediction } \\
\hline & & UXO & Partial & Base & Clutter & Scrap & Empty \\
\hline \multirow{6}{*}{ Ground truth } & UXO & 34 & 0 & 0 & 0 & 0 & 0 \\
\hline & Partial & 5 & 17 & 0 & 1 & 0 & 0 \\
\hline & Base & 0 & 0 & 39 & 0 & 1 & 0 \\
\hline & Clutter & 0 & 0 & 4 & 15 & 5 & 1 \\
\hline & Scrap & 2 & 1 & 3 & 5 & 11 & 0 \\
\hline & Empty & 1 & 1 & 2 & 2 & 0 & 0 \\
\hline
\end{tabular}

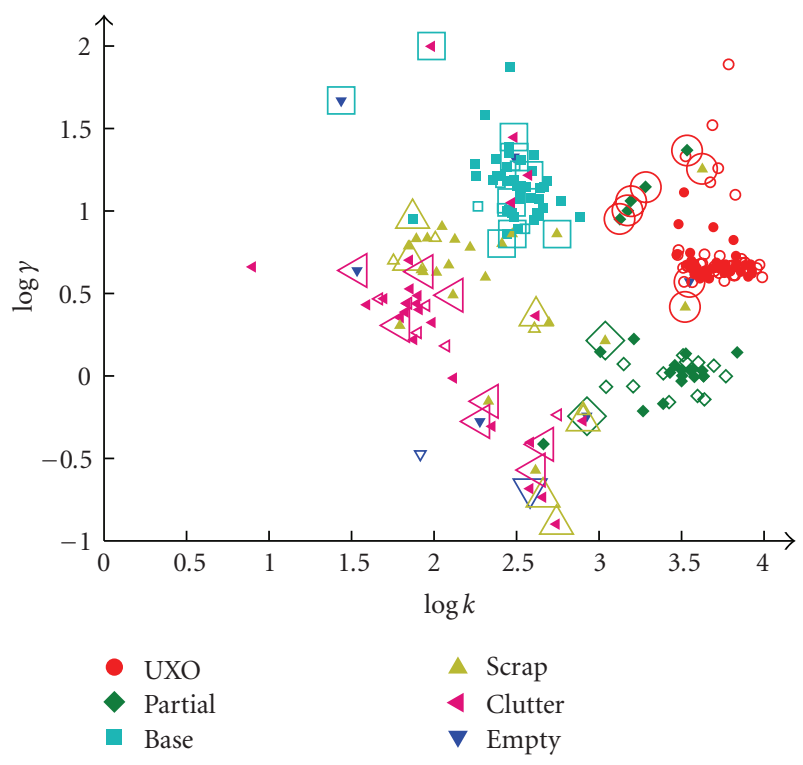

Figure 7: Result of the SVM classification for the Camp Sibert anomalies using the logarithms of the Pasion-Oldenburg parameters $k$ and $\gamma$. The SVM here has a capacity $C=9$. The small markers denote the ground truth for both training (hollow) and testing (solid) cells. The larger markers show the wrong SVM predictions.

It is helpful and straightforward to increase the dimensionality of the feature space. Figure 9 shows the discrimination obtained by running the SVM using all three PasionOldenburg features. The capacity $C=9$ here, and increasing it changes the results only slightly. The number of false alarms increases: we get the same two pieces of scrap from before, and now a few of the partial mortars are identified as UXO by the algorithm, due in part to the small range of $\beta$

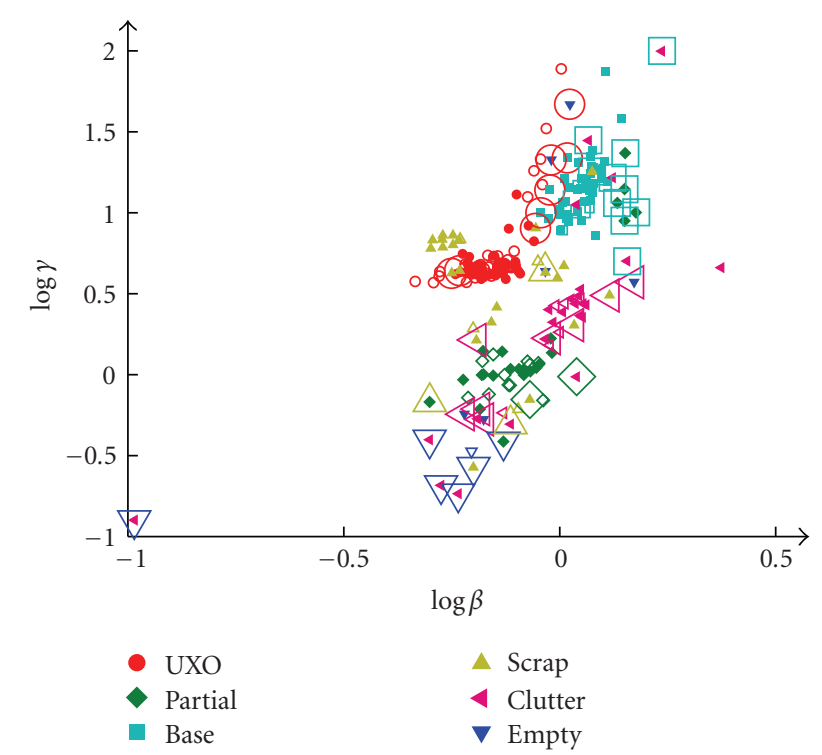

FIGURE 8: Result of the SVM classification for the Camp Sibert anomalies using the logarithms of the Pasion-Oldenburg parameters $\beta$ and $\gamma$. The SVM capacity $C=10^{5}$. The small markers denote the ground truth for both training (hollow) and testing (solid) cells. The larger markers highlight the wrong predictions made by the SVM.

and in part to the large gap between the UXO and the other anomalies, clearly visible in the figure, which again calls out for more and more-diverse training information.

Finally, it is possible to dispense with the PasionOldenburg model altogether and run an SVM using the "raw" $Q(t)$ as input. The feature space has dimensionality $\Delta=25$. We scale the values by $Q\left(t_{1}\right)$ and take the logarithm. We find $C=20$ to be the optimal value. Table 4 shows 
TABLE 3: SVM classification of Camp Sibert anomalies using $\gamma$ and $\beta$ with $C=10^{5}$.

\begin{tabular}{|c|c|c|c|c|c|c|c|}
\hline \multirow{2}{*}{\multicolumn{2}{|c|}{$\gamma, \beta ; C=10^{5}$}} & \multicolumn{6}{|c|}{ SVM prediction } \\
\hline & & UXO & Partial & Base & Clutter & Scrap & Empty \\
\hline \multirow{6}{*}{ Ground truth } & UXO & 34 & 0 & 0 & 0 & 0 & 0 \\
\hline & Partial & 0 & 14 & 5 & 2 & 1 & 1 \\
\hline & Base & 3 & 0 & 37 & 0 & 0 & 0 \\
\hline & Clutter & 0 & 1 & 5 & 14 & 1 & 4 \\
\hline & Scrap & 3 & 1 & 1 & 3 & 13 & 1 \\
\hline & Empty & 2 & 0 & 0 & 3 & 1 & 0 \\
\hline
\end{tabular}

TABLE 4: SVM classification of Camp Sibert anomalies using all $Q$ values (scaled by $Q\left(t_{1}\right)$ ) with $C=20$.

\begin{tabular}{cccccccc}
\hline \multirow{2}{*}{$\mathrm{Q} / \mathrm{Q}\left(t_{1}\right) ; C=20$} & \multicolumn{6}{c}{ SVM prediction } \\
\multirow{6}{*}{ Ground truth } & UXO & Partial & Base & Clutter & Scrap & Empty \\
\hline \multirow{6}{*}{} & UXO & 34 & 0 & 0 & 0 & 0 & 0 \\
& Partial & 0 & 15 & 0 & 7 & 1 & 0 \\
& Base & 3 & 0 & 34 & 3 & 0 & 0 \\
& Clutter & 0 & 2 & 3 & 14 & 4 & 2 \\
& Scrap & 3 & 1 & 3 & 3 & 12 & 0 \\
\hline
\end{tabular}

TABLE 5: SVM classification of Camp Sibert anomalies using all $Q$ values (unscaled) with $C=1$.

\begin{tabular}{cccccccc}
\hline \multirow{3}{*}{ All Q; $C=1$} & & \multicolumn{2}{c}{ SVM prediction } \\
\multirow{6}{*}{ Ground truth } & UXO & Partial & Base & Clutter & Scrap & Empty \\
\hline \multirow{6}{*}{} & UXO & 34 & 0 & 0 & 0 & 0 & 0 \\
& Partial & 5 & 17 & 0 & 1 & 0 & 0 \\
& Base & 0 & 0 & 39 & 0 & 1 & 0 \\
& Clutter & 0 & 2 & 4 & 18 & 1 & 0 \\
& Scrap & 2 & 2 & 3 & 2 & 13 & 0 \\
\hline
\end{tabular}

the results. The performance is slightly inferior to that of $R$ versus $k$; the usual two false alarms are there, along with a few new ones. All the UXO are identified correctly. We can also use the logarithm of $Q$ without any scaling (though the SVM internally rescales the feature space to $[0,1]^{\Delta}$ ). A capacity $C=1$ suffices here. The results appear on Table 5 . All dangerous items are once more identified as such.

\section{Conclusion}

In this paper, we have applied the NSMS model to EM63 Camp Sibert discrimination data sets. First the locations of the objects were inverted for by the fast and accurate dipole-inspired HAP method. Subsequently, each anomaly was characterized at each time channel through its total NSMS strength. Discrete intrinsic features were selected and extracted for each object using the Pasion-Oldenburg decay law and then used as input for a support vector machine that classified the items.
Our study reveals that the ratio of an object's late response to its early response can be used as a robust discriminator when combined with the Pasion-Oldenburg amplitude $k$. Other mixtures of these parameters also result in good classifiers. Moreover, we can use $Q$ directly, completely obviating the need for the Pasion-Oldenburg fit. In each case, the classifier runs by itself and does not require any human intervention. The SVM can be trained very quickly, even when the feature space has more than 20 dimensions, and it is a simple matter to add more training data on-the-fly. It is also possible to use already processed data to classify examples as yet unseen.

We should stress that none of our classifications yielded false negatives: all UXO were identified correctly in every instance. (This is due in part to the clean, UXO-intensive training data provided by the examiners and may change under different conditions.) The number of false alarms (false positives) varies with the classification features, but is in general low and can be as low as 2 out of 36 reported positives. Figures 6 and 5 show, among others, how these 


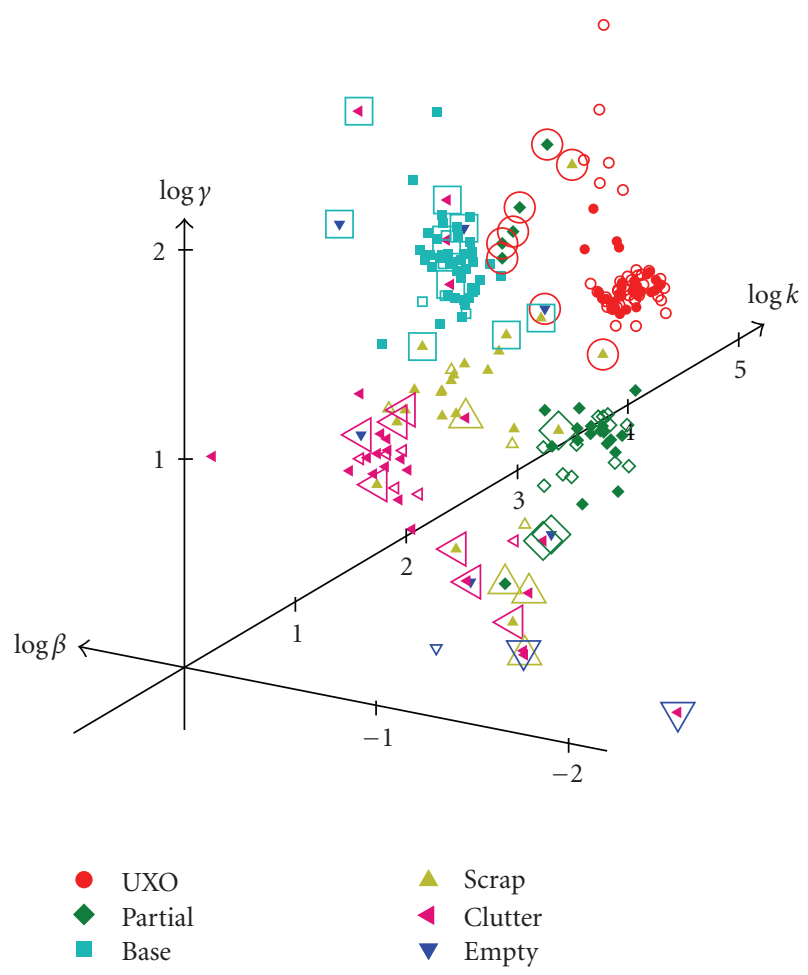

FIgURE 9: SVM classification of the Camp Sibert anomalies using the logarithms of $k, \beta$, and $\gamma$. The SVM has $C=9$. The small markers denote the ground truth for both training and testing cells. The larger markers highlight the cases where there is a disagreement between the ground truth and the SVM prediction.

false alarms come to be: some of the clutter items have a response that closely resembles that of UXO. While this will inevitably arise, it may still be possible to make the SVM more effective-and perhaps get close to reaching $100 \%$ accuracy-by including some of these refractory cases during the training. That said, there will certainly be cases in the field where the nonuniqueness inherent to noisy inverse scattering problems will cause the whole procedure to fail and yield dubious estimates. In those cases it will be necessary to assume the target is dangerous and dig it out.

In a completely realistic situation, where in principle no training data are given and the ground truth can be learned only as the anomalies are excavated, one can never be sure that the data already labeled constitute a representative sample containing enough of both dangerous and innocuous items. This difficulty is mitigated by two facts: (1) usually at the outset we have some idea of the kind of UXO present in the field and (2) the (usually great) majority of detected anomalies will not be UXO and thus random digging will produce a varied sampling of the clutter present. Methods involving semisupervised learning exploit this gradual revealing of the truth and have been found to perform better at UXO discrimination than supervised learning methods like SVM when starting from the point dipole model $[35,36]$. (Active learning methods, which try to infer which anomalies would contain the most useful information and could thus serve to guide the anomaly unveiling, show further, though fairly minor, improvement.) Combining this more powerful learning procedure with the excellent performance of the HAP/NSMS method may enhance the discrimination protocol and should be the subject of further research.

In summary, the results presented here show that our search and characterization procedure, whose effectiveness is apparent from several recent studies $[3,4,37,38]$, can be combined with an SVM classifier to produce a UXO discrimination system capable of correctly singling out dangerous items from among munitions-related debris and other natural and artificial clutter. In future investigations, we will continue to hone these algorithms and use them on other blind tests, including some already carried out in saltwater instead of soil.

\section{Acknowledgment}

This work was supported by the Strategic Environmental Research and Development Program through Grants no. MM-1572 and no. MM-1573.

\section{References}

[1] J. Byrnes, Ed., Unexploded Ordnance Detection and Mitigation, NATO Science for Peace and Security Series B: Physics and Biophysics, Springer, Dordrecht, The Netherlands, 2009.

[2] J. D. McNeill and M. Bosnar, "Application of TDEM techniques to metal detection and discrimination: a case history with the new Geonics EM-63 fully time-domain metal detector," Tech. Rep. TN-32, Geonics LTD, Mississauga, Canada, 2000, http://www.geonics.com/.

[3] F. Shubitidze, J. P. Fernández, B. E. Barrowes, I. Shamatava, and K. O'Neill, "Normalized surface magnetic source model applied to camp sibert data: discrimination studies," in Proceedings of the Applied Computational Electromagnetics Symposium (ACES '09), Monterey, Calif, USA, March 2009.

[4] F. Shubitidze, J. P. Fernández, I. Shamatava, L. R. Pasion, B. E. Barrowes, and K. O’Neill, “Application of the normalized surface magnetic source model to a blind unexploded ordnance discrimination test," Applied Computational Electromagnetics Society Journal, vol. 25, no. 1, pp. 89-98, 2010.

[5] C.-C. Chang and C.-J. Lin, "LIBSVM: a library for support vector machines," 2009, http://www.csie.ntu.edu.tw/ cjlin/libsvm/.

[6] B. E. Boser, I. M. Guyon, and V. N. Vapnik, "A training algorithm for optimal margin classifiers," in Proceedings of Annual 5th ACM Workshop on Computational Learning Theory, D. Haussler, Ed., pp. 144-152, ACM Press, London, UK, 1992.

[7] V. N. Vapnik, "An overview of statistical learning theory," IEEE Transactions on Neural Networks, vol. 10, no. 5, pp. 988-999, 1999.

[8] V. Cherkassky and F. Mulier, Learning from Data: Concepts, Theory, and Methods, John Wiley \& Sons, New York, NY, USA, 1998.

[9] C. Cortes and V. Vapnik, "Support-vector networks," Machine Learning, vol. 20, no. 3, pp. 273-297, 1995. 
[10] A. J. Smola and B. Schölkopf, "A tutorial on support vector regression," Statistics and Computing, vol. 14, no. 3, pp. 199222, 2004.

[11] C.-W. Hsu and C.-J. Lin, "A comparison of methods for multiclass support vector machines," IEEE Transactions on Neural Networks, vol. 13, no. 2, pp. 415-425, 2002.

[12] Y. Zhang, L. Collins, H. Yu, C. E. Baum, and L. Carin, "Sensing of unexploded ordnance with magnetometer and induction data: theory and signal processing," IEEE Transactions on Geoscience and Remote Sensing, vol. 41, no. 5, part 1, pp. 10051015, 2003.

[13] J. P. Fernández, B. Barrowes, K. O’Neill, et al., "Evaluation of SVM classification of metallic objects based on a magneticdipole representation," in Detection and Remediation Technologies for Mines and Minelike Targets XI, J. T. Broach, R. S. Harmon, and J. H. Holloway Jr., Eds., vol. 6217 of Proceedings of SPIE, Bellingham, Wash, USA, April 2006.

[14] X. Chen, Inverse problems in electromagnetics, Ph.D. dissertation, Massachusetts Institute of Technology, Cambridge, Mass, USA, June 2005.

[15] B. Zhang, K. O’Neill, J. A. Kong, and T. M. Grzegorczyk, "Support vector machine and neural network classification of metallic objects using coefficients of the spheroidal MQS response modes," IEEE Transactions on Geoscience and Remote Sensing, vol. 46, no. 1, pp. 159-171, 2008.

[16] J. P. Fernández, K. Sun, B. Barrowes, et al., "Inferring the location of buried UXO using a support vector machine," in Detection and Remediation Technologies for Mines and Minelike Targets XII, R. S. Harmon, J. T. Broach, and J. H. Holloway Jr., Eds., vol. 6553 of Proceedings of SPIE, pp. 65530B.1-65530B.9, Bellingham, Wash, USA, April 2007.

[17] A. Massa, A. Boni, and M. Donelli, "A classification approach based on SVM for electromagnetic subsurface sensing," IEEE Transactions on Geoscience and Remote Sensing, vol. 43, no. 9, pp. 2084-2093, 2005.

[18] E. Bermani, A. Boni, S. Caorsi, and A. Massa, "An innovative real-time technique for buried object detection," IEEE Transactions on Geoscience and Remote Sensing, vol. 41, no. 4, pp. 927-931, 2003.

[19] S. Caorsi, D. Anguita, E. Bermani, A. Boni, M. Donelli, and A. Massa, "A comparative study of NN and SVM-based electromagnetic inverse scattering approaches to on-line detection of buried objects," Applied Computational Electromagnetics Society Journal, vol. 18, no. 2, pp. 65-75, 2003.

[20] A. B. Tarokh, E. L. Miller, I. J. Won, and H. Huang, "Statistical classification of buried objects from spatially sampled time or frequency domain electromagnetic induction data," Radio Science, vol. 39, no. 4, Article ID RS4S05, 2004.

[21] A. Aliamiri, J. Stalnaker, and E. L. Miller, "Statistical classification of buried unexploded ordnance using nonparametric prior models," IEEE Transactions on Geoscience and Remote Sensing, vol. 45, no. 9, pp. 2794-2806, 2007.

[22] I. Shamatava, F. Shubitidze, B. Barrowes, J. P. Fernández, L. R. Pasion, and K. O'Neill, “Applying the physically complete EMI models to the ESTCP camp sibert pilot study EM-63 data," in Detection and Sensing of Mines, Explosive Objects, and Obscured Targets XIV, R. S. Harmon, J. T. Broach, and J. H. Holloway Jr., Eds., vol. 7303 of Proceedings of SPIE, pp. 73030O1-73030O11, Bellingham, Wash, USA, May 2009.

[23] F. Shubitidze, D. Karkashadze, B. Barrowes, I. Shamatava, and K. O’Neill, “A new physics-based approach for estimating a buried object's location, orientation and magnetic polarization from EMI data," Journal of Environmental and Engineering Geophysics, vol. 13, no. 3, pp. 115-130, 2008.
[24] F. Shubitidze, K. O’Neill, B. E. Barrowes, et al., “Application of the normalized surface magnetic charge model to UXO discrimination in cases with overlapping signals," Journal of Applied Geophysics, vol. 61, no. 3-4, pp. 292-303, 2007.

[25] L. R. Pasion and D. W. Oldenburg, "A discrimination algorithm for UXO using time domain electromagnetics," Journal of Environmental \& Engineering Geophysics, vol. 6, pp. 91-102, 2001.

[26] A. G. Kyurkchan, B. Y. Sternin, and V. E. Shatalov, "Singularities of continuation of wave fields," Physics-Uspekhi, vol. 39, no. 12, pp. 1221-1242, 1996.

[27] R. Zaridze, G. Bit-Babik, K. Tavzarashvili, D. P. Economou, and N. K. Uzunoglu, "Wave field singularity aspects in largesize scatterers and inverse problems," IEEE Transactions on Antennas and Propagation, vol. 50, no. 1, pp. 50-58, 2002.

[28] M. A. Aizerman, E. M. Braverman, and L. I. Rozonoer, "Theoretical foundations of the potential function method in pattern recognition learning," Automation and Remote Control, vol. 25, pp. 821-837, 1964.

[29] C. J. C. Burges, "A tutorial on support vector machines for pattern recognition," Data Mining and Knowledge Discovery, vol. 2, no. 2, pp. 121-167, 1998.

[30] O. L. Mangasarian and D. R. Musicant, "Lagrangian support vector machines," Journal of Machine Learning Research, vol. 1, no. 3, pp. 161-177, 2001.

[31] J. Mercer, "Functions of positive and negative type and their connection with the theory of integral equations," Philosophical transactions of the Royal Society of London. Series A, vol. 209, pp. 415-446, 1909.

[32] E. Bermani, A. Boni, A. Kerhet, and A. Massa, "Kernels evaluation of SVM-based estimators for inverse scattering problems," The phenomenon of resonance radiation of electromagnetic Research, vol. 53, pp. 167-188, 2005.

[33] N. Cristianini and J. Shawe-Taylor, An Introduction to Support Vector Machines and other Kernel-Based Learning Methods, Cambridge University Press, Cambridge, UK, 2000.

[34] U. Kreßel, "Pairwise classification and support vector machines," in Advances in Kernel Methods: Support Vector Learning, B. Schölkopf, J. C. Burges, and A. J. Smola, Eds., pp. 268-255, MIT Press, Cambridge, Mass, USA, 1999.

[35] Y. Zhang, X. Liao, and L. Carin, "Detection of buried targets via active selection of labeled data: application to sensing subsurface UXO," IEEE Transactions on Geoscience and Remote Sensing, vol. 42, no. 11, pp. 2535-2543, 2004.

[36] Q. Liu, X. Liao, and L. Carin, "Detection of unexploded ordnance via efficient semisupervised and active learning," IEEE Transactions on Geoscience and Remote Sensing, vol. 46, no. 9, pp. 2558-2567, 2008.

[37] F. Shubitidze, B. Barrowes, I. Shamatava, J. P. Fernández, and K. O’Neill, "APG UXO discrimination studies using advanced EMI models and TEMTADS data," in Detection and Sensing of Mines, Explosive Objects, and Obscured Targets XIV, R. S. Harmon, J. T. Broach, and J. H. Holloway Jr., Eds., vol. 7303 of Proceedings of SPIE, Bellingham, Wash, USA, May 2009.

[38] I. Shamatava, F. Shubitidze, B. Barrowes, J. P. Fernández, and K. O’Neill, "Physically complete model applied to BUD timedomain EMI data," in Detection and Sensing of Mines, Explosive Objects, and Obscured Targets XIV, R. S. Harmon, J. T. Broach, and J. H. Holloway Jr., Eds., vol. 7303 of Proceedings of SPIE, pp. 73030N1-73030N-9, Bellingham, Wash, USA, May 2009. 